RESUMEN: Dos de los temas recurrentes en la obra de Edgar Allan Poe (1809-1849) son el origen y el orden del mundo, a los que este autor consideraba expresiones de una realidad espiritual superior, y para el abordaje de los cuales escribió textos que iban desde la filosofía del mobiliario y el paisajismo hasta la cosmología, en un intento por explicar el sentido del mundo físico para los seres humanos, así como su impacto en ellos a partir de formas, magnitudes y distribución.

PALABRAS CLAVE: cosmología, intuición, paisajismo, trascendentalismo.

\section{EL ORDEN DEL CAOS: NATURALEZA, ORDEN Y REGULARIDAD DEL MUNDO SEGÚN EDGAR ALLAN POE Javier Ayala Calderón*} Javier Ayala Calderón

THE ORDER OF CHAOS: NATURE, ORDER AND REGULARITY OF THE WORLD ACCORDING to Edgar Allan PoE

ABSTRACT: Two of the recurrent topics in Edgar Allan Poe's (1809-1849) work are the origin and order of the world, which this author considered expressions of a superior spiritual reality, and for the approach of which he wrote some texts that went from the philosophy of furniture and landscaping to cosmology in an attempt to explain the sense of the physical world for human beings, as well as its impact on them from forms, magnitudes and distribution.

KEYWORDS: cosmology, intuition, landscaping, transcendentalism.

* Departamento de Historia, Universidad de Guanajuato. 


\author{
EL ORDEN DEL CAOS: \\ NATURALEZA, ORDEN Y \\ REGULARIDAD DEL MUNDO \\ SEGÚN EDGAR ALLAN POE
}

\title{
Introducción: Edgar Allan Poe
}

A Edgar Allan Poe (Boston, 19 de enero de 1809 - Baltimore, 7 de octubre de 1849) se le ha reconocido universalmente, una y otra vez, por su obra narrativa y poética y se le considera un maestro en el tratamiento del terrory el misterio. Sin embargo, como afirma Margarita Rigal Aragón refiriéndose "sobre todo, aunque no únicamente" a sus cuentos "grotescos" (es decir, cómico-satíricos), ${ }^{1}$ muy pocas veces, y con limitaciones, se han estudiado aquellas obras de Poe que recogen sus inquietudes de actualidad acerca de la política, la vida

${ }^{1}$ Margarita Rigal Aragón, "Los cuentos 'relegados' de Edgar Allan Poe", REDEN 19-20 (2000): 87. social, la literatura, la filosofía y la ciencia de Estados Unidos de la primera mitad del siglo XIX, temas por los que sentía un vivo interés y que proyecta con gran amenidad y belleza en sus escritos, pero que, debido a su fugacidad y su menor universalidad, eluden y cansan al lector moderno, alejado de la atmósfera y los intereses en que fueron producidos. ${ }^{2}$ Por consiguiente, para poder comprender mucho de lo plasmado por Poe en dichos trabajos, primero hay que familiarizarse con la época y las ideas imperantes.

${ }^{2}$ Ibid., 86. 
Dos temas predilectos de Poe, y que compartían muchos de sus contemporáneos, fueron el origen y el orden del mundo, así como la necesidad de encontrar un método adecuado para conocerlos. Por supuesto, en los Estados Unidos de principios del siglo XIX el científico no era el único sistema de pensamiento con el que se trataba de dar cuenta del cosmos, sino que aun dentro de los movimientos religiosos y filosóficos dominantes hubo intentos de comprenderlo como expresión de una realidad superior, y es ahí donde podemos insertar algunos de los trabajos más interesantes, pero menos conocidos de Poe ("Filosofía del mobiliario", 1840; "El dominio de Arnheim", 1847; ${ }^{3}$ Eureka, 1848, y “El cottage de Landor", 1849), los cuales abordaremos enseguida junto con otras obras complementarias, para mostrar cómo la literatura de este personaje - usualmente calificado de fantástico (y que así lo parece sin dudas a la distancia) - en su momento fue también un reflejo de corrientes de pensamiento dedicadas a comprender la realidad del universo por vías distintas a las de la ciencia de su tiempo.

3 "El dominio de Arnheim" tiene una versión ligeramente más breve de 1842 que se conoce como "El paisaje del jardín".

\section{El mundo y la intuición como método de acercamiento}

La etapa que a Edgar Allan Poe le tocó vivir en Estados Unidos fue de grandes avances tecnológicos y de consolidación económica. Muchas ciencias, como la química y la física, avanzaban cada vez más aprisa y con mayor certidumbre, mientras que habían aparecido nuevas disciplinas, como la geología, la biología, la zoología y la antropología en su sentido moderno, con un empuje proporcional a la capacidad que las nuevas herramientas y métodos de trabajo les daban para demostrar sus descubrimientos.

No obstante, mientras Humboldt y Bonpland medían el mundo para tener una idea clara de su conformación física y los estudios de la electricidad y el electromagnetismo de Galvani, Ampere y Faraday daban pie a nuevas interpretaciones de la naturaleza de la vida, paralelamente las antiguas formas de ver continuaban aferrándose a sus tradiciones o transformándose lentamente y a regañadientes en su intento de adaptarse a las nuevas circunstancias. De esta manera, para la última década de su vida, Poe se encontraba inmerso en un ambiente de cambios religiosos y filosóficos que influían notablemente en los intelectuales estadounidenses en la forma del llamado trascendentalismo. 
El trascendentalismo fue un movimiento filosófico, político y literario que había comenzado como parte de una reforma unitaria de las iglesias congregacionalistas de Estados Unidos como oposición al trinitarismo tradicional, solo que, llevando esta unificación al extremo, y por la influencia del romanticismo alemán, el trascendentalismo concluyó en un monismo que sostenía la unidad del mundo con Dios mediante una explicación inmanentista. Para los creyentes de esta doctrina, el alma de cada individuo era idéntica al alma del mundo $\mathrm{y}$, por lo tanto, contenía igualmente vestigios de la divinidad. ${ }^{4}$

Mientras que en el unitarismo, como herencia de la Ilustración del siglo anterior, se aceptaba que la revelación debía ser racionalmente comprensible para los creyentes y, por lo tanto, que la razón era una herramienta fundamental para el cristiano, ${ }^{5}$ los trascendentalistas, impregnados del romanticismo de su época, entendían la razón en un sentido distinto a la mera facultad del hombre para realizar actividades mentales, y la veían como una forma más alta, "intuitiva, experiencial y apasionada" de abordar la

${ }^{4}$ James D. Hart, The Oxford companion to American literature (Nueva York: Oxford University Press, 1980), 772-773.

${ }^{5}$ Donna M. Campbell, “American transcendentalism”, en Literary movements, Department of English, Washington State University, http://public. wsu.edu/ campbelld/amlit/amtrans.htm. fe y todo lo que con ella se relacionara. ${ }^{6}$ Por lo mismo, mientras que el unitarismo proclamaba que las Escrituras eran la prueba de la verdad de la fe apoyándose en razonamientos teológicos, los trascendentalistas estaban convencidos de que no había pruebas empíricas satisfactorias para las creencias religiosas, ${ }^{7}$ sino que era necesario conformarse con una intuición de Dios por medio de la contemplación y experiencia de sus obras en la naturaleza.

La gran notoriedad de este movimiento enervaba a Poe, que no creía en la facilidad simplista del recurso enunciado como método de conocimiento. Sin embargo, como expresó a su amigo Thomas Holley Chivers en carta del 10 de junio de 1844, su desdén no iba en contra del trascendentalismo como tal, en el que al fin y al cabo no veía más que un misticismo optimista practicado por personas instruidas y bien intencionadas, sino solo contra: "los pretenciosos y los sofistas entre ellos" (como conceptuaba concretamente al entonces popular escritor y conferencista Ralph Waldo Emerson, cabecilla del Trascendental Club, formado en Boston en 1836 con un grupo de escritores

\footnotetext{
${ }^{6}$ Jone Johnson Lewis, "Trascendentalism", http://www.transcendentalists.com/what.htm.

${ }^{7}$ Russell Goodman, "Trascendentalism”, en The Stanford encyclopedia of philosophy, http:// plato.stanford.edu/archives/fall2015/entries/transcendentalism/.
} 
como Henry David Thoreau y Louise May Alcott), quienes creían encontrar en esta doctrina una manera de ponerse en contacto con lo absoluto espiritual en un alarde de misticismo por el misticismo en sí, sin que sus "desvaríos" trajeran ningún beneficio a nadie. ${ }^{8}$ Esos desvaríos los llevaban en casos extremos a huir de las grandes ciudades para refugiarse en lo profundo de los bosques vírgenes, en pos de un ambiente propicio para el contacto individual con Dios, como hizo Henry David Thoreau en las cercanías del lago Walden (en Concord, Massachusetts) o fundando pequeñas aldeas utópicas en las campiñas como Brook Farm y Fruitlands en la década de $1840 .{ }^{9}$

A pesar de las acerbas críticas que dirigía a este movimiento, las teorías personales de Poe acerca del mundo y la manera de experimentarlo tenían ciertos puntos de contacto con las doctrinas trascendentalistas. Dehecho, es posible que haya sido precisamente ese parecido lo que lo llevó a escribir a su amigo Chivers que, pese a tales puntos en común, su fe era exclusivamente suya, indicándole, además, que la encontraría algo más detallada

${ }^{8}$ Edgar Allan Poe, "Capítulo sobre autografia", en Miscelánea, trad. por Gerardo Bensi et al. (Buenos Aires: Claridad, 2006), 207-208.

${ }^{9}$ Richard Francis, Transcendental utopias: Individual and community at Brook Farm, Fruitlands and Walden (Ithaca/Londres: Cornell University Press, 1997). en el cuento ("artículo", como él lo llama en su misiva) "Revelación mesmérica", que estaba a punto de aparecer para agosto o septiembre de aquel año en la Columbian Magazine, la cual se publicaba entonces en Nueva York. ${ }^{10}$

En ese texto, el moribundo señor Vankirk caracteriza a Dios como "la materia imparticulada", última o indivisa que penetra todas las cosas y las impulsa, de tal manera que es todas las cosas en sí misma, pero que al mismo tiempo tiene características particulares ajenas a su creación que lo diferencia de esta. ${ }^{11}$ Con lo anterior, Poe estaría abrazando una especie de panenteísmo que afirmaba no la identidad entre Dios y el mundo (como el panteísmo trascendentalista), sino a la vez la inmanencia y la transcendencia de Dios con respecto a un mundo que engloba, pero en el cual no se agota. Esta cosmovisión, explícitamente presentada como propia por el autor, no se repite en otros escritos fantásticos de Poe y por supuesto no tendría por qué hacerlo, toda vez que no podemos presumir entre ellos una congruencia filosófica sin obviar el riesgo siempre presente de confundir

${ }^{10}$ Carta a Thomas Holley Chivers, Nueva York, 10 de julio de 1844, en The collected letters of Edgar Allan Poe, ed. por John Ward Ostrom (Nueva York: The Gordian Press, 1966), I, 259.

${ }^{11}$ Edgar Allan Poe, "Mesmeric revelation", en The complete tales and poems of Edgar Allan Poe (Nueva York: Race Point Publishing, 2014), 45. 
las ideas del narrador con las de sus personajes. ${ }^{12}$ En cambio, sí pueden detectarse en algunos pasajes de su ensayo o poema en prosa Eureka (1848), que (pese a la posibilidad de que se trate de un engaño como "El camelo del globo"), ${ }^{13}$ explícitamente pretendía contener sus creencias acerca del cosmos. En Eureka volvemos a encontrar afirmaciones como las de que la primera acción de Dios fue crear la materia en su estado más puro de simplicidad, ${ }^{14}$ que tal acción fue más bien una concepción o idea, ${ }^{15}$ que a partir de eso "God [...] became all things at once, through dint of his volition, while all things were thus constituted a portion of God" "16 $y$, por consiguiente, también que "each soul is, in part, its own God". ${ }^{17}$ Pero solo en parte, pues "that nature and the God of nature are distinct, no thinking being can long doubt". ${ }^{18}$

Por otro lado, si bien Poe dudaba de los métodos místicos y románticos para establecer contacto con lo abso-

${ }^{12}$ David N. Stamos, Edgar Allan Poe, Eureka, and scientific imagination (Albany: State University of New York Press, 2017), 383.

${ }^{13}$ Relato de Poe que originalmente se presentó como una noticia verdadera en The New York Sun el 13 de abril de 1844. Peter Ackroyd. Poe. Una vida truncada, trad. por Bernardo Moreno Carrillo (Barcelona: Edhasa, 2009), 110-111.

${ }^{14}$ Edgar Allan Poe, Eureka. A prose poem (Nueva York: George P. Putnam, 1848), 29.

${ }^{15}$ Ibid., 62.

${ }^{16}$ Ibid., 81.

${ }^{17}$ Ibid., 141.

${ }^{18}$ Ibid., 80. luto por medio de la contemplación, tanto él como los trascendentalistas coincidían en el valor de la intuición. Para Poe no había ninguna oposición entre esta y la razón pues, pese a los exabruptos ocasionales de sus personajes al respecto, la intuición "is but the conviction arising from those inductions or deductions of which the processes are so shadowy as to escape our consciousness, elude our reason or defy our capacity of expression". ${ }^{19}$ En efecto, aunque los cuentos de raciocinio de Poe apelan constantemente al uso de la razón, el autor no duda en hacer simultáneamente la apología de la intuición como privilegio de todo hombre de genio, ${ }^{20} 10$ cual nos habla de las capacidades de por lo menos un sector de la humanidad para captar, no siempre necesariamente por medio del método científico, pero sí por encima del misticismo caprichoso, la lógica y la regularidad que se creía imperaba detrás del aparente caos del mundo y permitía su conocimiento.

$\mathrm{Y}$ fue precisamente a partir de esta intuición que Poe creyó haber encontrado la manera de explicar la contradicción que veía entre la esperada perfección de la naturaleza en el mundo como resultado de la presencia parcial de Dios en ella, en

\footnotetext{
${ }^{19}$ Carta a Charles F. Hoffman, 20 de septiembre de 1848, en The collected letters, 379-382.

${ }^{20}$ Edgar Allan Poe, "Un capítulo de sugerencias", en Miscelánea, 248-250.
} 
abierto contraste con la naturaleza virgen y anárquica que tanto atraía a los trascendentalistas, pero que para él no era más que un mero problema de orden y perspectiva.

\section{Orden y regularidad del mundo}

En efecto, para Poe el universo y la deidad que lo conformaba se manifestaban a ras de suelo como una belleza derivada del orden, la proporción y la regularidad, y donde estos no fueran evidentes, eso debía ser porque su visión resultaba superior a las capacidades sensoriales del hombre, no porque no existieran. En busca de este orden supremo, la obra de Edgar Allan Poe va desde los enunciados más abstractos y cosmológicos de Eureka hasta los más prácticos y materiales como los que vemos en los relatos "El dominio de Arnheim" (1847) y "El cottage de Landor" (1849), usualmente calificados como ensayos de jardinería, pero que en realidad tienen un fondo filosófico innegable disfrazado en ambos casos de viaje pintoresco y hasta fantástico.

En estos escritos, Poe invoca explícitamente los lineamientos marcados por los comentarios sobre los jardines ingleses ${ }^{21}$ tan populares en aquellos días en Inglaterra dentro

\footnotetext{
${ }^{21}$ Edgar Allan Poe, "Landor's Cottage", en The complete tales and poems, 540.
}

de la tradición de ensayistas como Alexander Pope (1688-1744), Joseph Addison(1672-1719), Horace Walpole (1717-1797) y otros, que procuraban darle a estos espacios cierto toque de tranquilizadora y confortable regularidad humana procurando no caer en los excesos del jardín francés de André Le Nôtre (1613-1700), jardinero de Luis XIV, cuando el absolutismo en aquellas tierras trataba de imponer el dominio irrestricto del hombre sobre la naturaleza con sus simétricos arabescos y sus repetitivas bordaduras de boj. Como explica Alain Roger:

Contrariamente a lo que pueda decirse, o creerse, la reacción a las simetrías francesas no se traduce por una naturalización del paisaje, sino por una pictorización del país. Cuando Joseph Addison, en el Spectator del 25 de junio de 1712, se rebela contra la manía de mutilar los árboles para reducirlos a "conos, globos y pirámides", este proceso de geometrización traduce únicamente un cambio de referencia artística: el modelo arquitectónico, representado por Le Nôtre, se sustituye por un modelo pictórico. ${ }^{22}$

Ajeno pues a los excesos franceses, el jardín paisajista inglés hacía una

${ }^{22}$ Alain Roger, Breve tratado del paisaje, trad. por Maysi Veuthey (Madrid: Biblioteca Nueva, 2014), 44. 
alianza con la naturaleza en busca de una armonía en la que cada una de las partes respetaba a la otra y se exaltaban los ideales de libertad y de fraternidad bajo la guía del conocimiento y de la razón, ${ }^{23}$ una idea exitosa y romántica entre los grupos ingleses liberales del siglo XVIII, pero solo parcialmente asimilada por los trascendentalistas estadounidenses del XIX, más bien dados a la búsqueda de paisajes verdaderamente naturales y umbríos en los que intuían la presencia de lo divino y a los que juzgaban revitalizantes y capaces de confortar el alma en la soledad. ${ }^{24}$

Por su parte, inconforme con las imperfecciones del mundo físico, pero igualmente descontento con la simetría repetitiva del jardín francés, Poe opinaba que "in the most enchanting of natural landscapes there will always be found a defect or an excess - many excesses and defects. While the component parts may defy, individually, the highest skill of the artist, the arrangement of these parts will always be susceptible of improvement". ${ }^{25}$ Por eso, porque "no such combination of scenery exists in nature as the painter

${ }^{23}$ El espiritu del lugar. Jardin y paisaje en la Inglaterra moderna, ed. por Paula Martín Salván (Madrid: Abada, 2006), 32.

${ }^{24}$ Marta Nieto Bedoya, "La lectura oculta del jardín paisajista", en El lenguaje oculto del jardín: Jardín y metáfora, ed. por Carmen Añón Feliú (Madrid: Editorial Complutense, 1996), 122.

${ }^{25}$ Edgar Allan Poe, "The domain of Arnheim", en The complete tales and poems, 531. of genius may produce", ${ }^{26}$ para Poe, como para Joseph Adisson (el famoso defensor del jardín inglés del siglo XVIII), la belleza natural era más bella en la medida que más se parecía a la obra de arte. ${ }^{27}$ Solo que mientras Adisson destacaba su aspecto natural, Poe ponía el énfasis en la participación humana y francamente matemática en la concepción del jardín, por lo que este no debía ser de tal forma que recordara la belleza original del campo, sino que resultara "partly pleasing to the eye, by the show of order and design, and partly moral", ${ }^{28}$ por lo que respectaba al sentimiento del interés humano puesto en ello y las ideas que implicaba.

El desarrollo de esta teoría es lo que podemos ver en los "ensayos de jardinería" arriba referidos.

Aparentemente "El cottage de Landor" se encuentra inspirado en el cottage de Fordham, una propiedad rural de los condados fluviales de Nueva York, ${ }^{29}$ veinticinco kilómetros al norte de la ciudad, a donde los Poe llegaron como inquilinos en 1846 buscando demasiado tarde el aire fresco que le devolviera la salud a Virginia,

${ }^{26}$ Ibid.

${ }^{27}$ Joseph Addison. "Los placeres de la imaginación”, en El espiritu del lugar, p. 51. Aunque en el texto del artículo citado a Addison se le llame John, la cubierta consigna correctamente su nombre. Véase: Poe, "The domain of Arnheim", 531-532.

${ }^{28}$ Ibid., 533.

${ }^{29}$ Poe, "Landor's Cottage", 539. 
la tuberculosa prima y esposa del escritor. Aunque un contemporáneo suyo describe este lugar como un "descampado lleno de piedras, arbustos y cardos" con apenas alguna que otra casa de labranza en los alrededores, las ventanas frontales del edificio daban al valle del Hudson y desde ellas se disfrutaba una magnífica vista de su corriente, ${ }^{30}$ lo cual explica que Poe pudiera describirla como un paisaje ajardinado.

En este relato, a medida que el viajero narrador se aproxima al cottage, el camino lleno de rodaduras de coche y los árboles, en el estilo escuálido y plumoso del pintor Salvatore Rosa (1615-1673), ceden paso a un sendero cada vez más regular y una organización de la naturaleza más evidente, hasta que el viajero lanza una primera observación acerca de lo que está viendo, la cual coincide con el pensamiento del autor en su búsqueda del orden humano dentro del aparente desorden del mundo:

[A]n artist, and one with a most scrupulous eye for form, had superintended all these arrangements. The greatest care had been taken to preserve a due medium between the neat and graceful on the one hand, and the pittoresque, in the true sense of the Italian term, on the other. There were few straight, and no long

\footnotetext{
${ }^{30}$ Ackroyd. Poe. Una vida truncada, 112.
}

uninterrupted lines. The same effect of curvature or of color, appeared twice, usually, but not oftener, at any one point of view. Everywhere was variety in uniformity. ${ }^{31}$

Poco a poco la vegetación se vuelve menos salvaje, más suave y más graciosa, es decir, más a la medida del hombre culto (no del meramente animal), hasta llegar a una especie de anfiteatro rodeado por completo de escarpas pedregosas, pero cubierto de un césped "suave, espeso, aterciopelado y milagrosamente verde", 32 muy similar al que rodeaba el cottage de Poe, según una descripción hecha por la señora Mary Gove Nichols en $1863^{33}$ a raíz de una visita hecha en 1846 ("an acre or two ${ }^{34}$ of greensward, fenced in about the house, as smooth as velvet and as clean as the best kept carpet") ${ }^{35}$ y que ya desde la época de Francis Bacon (1561-1626) se consideraba placentero tanto por sus cualidades estéticas, como por la facilidad para trazar caminos en él. ${ }^{36}$

${ }^{31}$ Poe, "Landor's Cottage", 540.

${ }^{32} \mathrm{Ibid}$., 542.

${ }^{33}$ Arthur Hobson Quinn, Edgar Allan Poe: A critical biography (Nueva York/Londres: D. Appleton-Century Company Incorporated, 1941), 507.

${ }^{34}$ Menos de una hectárea (el acre equivale a 4046.86 metros cuadrados o 0.4 hectáreas).

${ }^{35}$ Mary Gove Nichols, "Carta", en The collected works of Edgar Allan Poe, ed. por T. O. Mabbott (Cambridge/Londres: The Belknap Press of Harvard University Press, 1978), III, 1326.

${ }^{36}$ Francis Bacon, "De los jardines", en El espiritu del lugar, 41. 
El anfiteatro del relato se encontraba surcado por un arroyuelo de "excesiva limpidez", bucólico trasunto del río Hudson, y en él pastaban una gran manada de borregos y tres ciervos domesticados: lo criado por el hombre y lo natural hecho a su medida, unos al lado de los otros en una amalgama suprema de belleza y rentabilidad, pues para la espiritualidad protestante el disfrute estético no se encuentra desligado del provecho. El espacio se transformaba así incorporando rebaños, arbolado y granjas a la naturaleza circundante haciendo rentable lo que es bello, "idea que evolucionará hasta hacer bello lo que era rentable con el tratamiento paisajista de las granjas y las explotaciones agrícolas", ${ }^{37}$ tal como ocurrió en la versión económicamente utilitaria del paisajismo en los libros de Andrew Jackson Downing (18151852) A treatise on the theory and practice of landscape gardening, adapted to North America (1841) y Cottage residences or, A series of designs for rural cottages and adapted to North America (1842) con los que este autor creía posible acabar con la rusticidad de la arquitectura rural de los Estados Unidos mejorando de paso la moral de sus habitantes por medio del decoro. Fueron textos ampliamente difundidos y seguramente conocidos por Poe, aunque no fuera más que

${ }^{37}$ Marta Nieto Bedoya, "La lectura oculta del jardín paisajista", 123. de oídas dada su labor como periodista y crítico literario.

$Y$ en efecto, la casa que se alza en aquel valle y que en la realidad había sido construida como tantas otras de su tiempo (del modo más exiguo como refugio contra las inclemencias del tiempo, un espacio apenas suficiente para comer, beber y dormir), ${ }^{38}$ es minuciosamente descrita no solo en su apariencia, sino incluso en sus medidas, su orientación y sus materiales como un lugar idílico que proyecta una sensación de correspondencia, vivacidad y utilidad. Todo en esa construcción es un paisaje, incluyendo a los habitantes de la casa y la casa misma, versión utilitaria de una de aquellas "locuras" o construcciones idealizadas tan comunes de los jardines ingleses, pues como Poe afirmaba, "una mezcla de puro arte en el marco de un jardín le añade gran belleza". ${ }^{39}$ Incluso en el moblaje de la casa el viajero encuentra motivos para alabar la regularidad y la congruencia, pues a pesar de su sobriedad y escasez, se podía notar que "the forms of all had evidently been designed by the same brain which planned 'the grounds"". ${ }^{40}$ Por supuesto, a unos jardines diseñados mental-

${ }^{38}$ Andrew Jackson Downing, "On the moral influence of good houses", Horticulturist 2, febrero de 1848, 345, https://web.archive.org/web/ 20100706032413/http://www.fandm.edu/x10620.

${ }^{39}$ Poe, "The domain of Arnheim", 533.

${ }^{40}$ Poe, "Landor's cottage", 547. 
mente correspondían unos muebles si no nada más imaginados, al menos sí artísticamente dotados de un halo romántico que los ennoblece ante el lector a pesar de su humildad. De hecho, y en congruencia con su filosofía del paisaje, Poe sostenía que la verdadera elegancia de una habitación es como un cuadro pintado antes que como la realidad de donde se toma:

We speak of the keeping of a room as we would of the keeping of a picture, for both the picture and the room are amenable to those undeviating principles which regulate all varieties of art; and very nearly the same laws by which we decide on the higher merits of a painting suffice for decision on the adjustment of a chamber. ${ }^{41}$

Poe escribió este párrafo nueve años antes de la publicación de "El cottage de Landor", en un texto todavía menos conocido llamado "La filosofía del mobiliario" (1840), pleno de ese tono cáustico que el autor solía utilizar cuando hablaba sobre la cultura en Estados Unidos. Al margen de su tono, esta obra habla de la decoración de interiores partiendo de una estética pictórica basada en principios geometrizantes y reglas matemáticas como una manera de moralizar a la sociedad estadounidense llevando al

${ }^{41}$ Edgar Allan Poe, "Philosophy of furniture", en The complete tales and poems, 406. interior de las casas los principios de la filosofía inglesa del paisaje arriba referida.

De esta forma, si Poe escribía complacido que en los alrededores del cottage de Landor había pocas líneas rectas y faltaban las líneas largas e ininterrumpidas, teniendo en cambio efectos de curvatura y color que aparecían sin cansadas repeticiones en cualquier punto de vista "dándole variedad a la uniformidad", ${ }^{42}$ justo lo contrario (incluso en el mismo orden), como en un espejo, criticaba ya en "La filosofía del mobiliario" hablando del moblaje de las casas estadounidenses de las clases acomodadas: "Very often the eye is offended by their inartistic arrangement. Straight lines are too prevalent, too uninterruptedly continued, or clumsily interrupted at right angles. If curved lines occur, they are repeated into unpleasant uniformity. Undue precision spoils the appearance of many a room".

Así pues, sin dejar de coincidir al menos parcialmente con ella, la casa descrita en "El cottage de Landor" es un edificio que corresponde más al ideal estético y espiritual expresado desde años atrás por Poe que con la sobria cabaña de Fordham, donde en 1847 murió finalmente su esposa y donde este autor pasó los últimos años de su vida.

\footnotetext{
${ }^{42}$ Poe, "Landor's cottage", 540.
} 


\section{La perspectiva perfecta}

En "El dominio de Arnheim", y pese a considerar que la naturaleza es soberana en sus formas, colores y "detalles", Poe no duda en afirmar nuevamente que la verdadera belleza del paisaje depende de las matemáticas, pues solo en ellas se sabe cuáles son las disposiciones de elementos que la constituyen. ${ }^{43}$ Por lo mismo, a pesar de que los múltiples meandros del río mencionado en este relato, así como el camino en "El cottage de Landor", tan sinuoso que en ningún momento podía prever su curso más allá de dos o tres pasos ${ }^{44}$ encarnaban la libertad y la naturalidad frente a las avenidas rectas y afectadas del jardín barroco, ${ }^{45}$ nos encontramos de cualquier manera ante la artificialidad enmascarada del jardín paisajista, con un mundo de regularidades que nos impacta por su carácter suprahumano. Así, con respecto a Arnheim, dice Poe:

The thought of nature still remained, but her character seemed to have undergone modification: there was a weird symmetry, a thrilling uniformity, a wizard propriety in these her works. Not a dead branch, not a withered leaf, not a stray pebble, not a patch of the brown earth, was any-

${ }^{43}$ Poe, "The domain of Arnheim", 531.

${ }^{44}$ Poe, "Landor's cottage", 540.

${ }^{45}$ Marta Nieto Bedoya, "La lectura oculta del jardín paisajista", 124. where visible. The crystal water welled up against the clean granite, or the unblemished moss, with a sharpness of outline that delighted while it bewildered the eye. ${ }^{46}$

La belleza depende aquí de la perfección de lo joven, lo sano y lo regular, pero esto no contradice el énfasis de Poe en la melancolía como un elemento "inseparablemente conectado con todas las más altas manifestaciones de verdadera belleza", según expone en su ensayo El principio poético (1848-1849, publicado póstumamente en 1850), ${ }^{47}$ pues incluso la más hermosa y juvenil escena puede ser portadora de una "disfrutable tristeza".

Y es precisamente en función de este último principio literario que la creación de un espacio de orden artificial perfecto, más que evocación de la naturaleza mejorada, sea en Poe imagen de un espacio sobrenatural, pues, lejos de ser un mero paraíso terrestre de donde se han desterrado la muerte y todos sus epifenómenos, resulta ser en diferentes niveles imagen de la tumba misma y símbolo del más allá. Basta con notar cómo el edificio construido en Arnheim se sostiene "como por milagro en el aire",

${ }^{46}$ Poe, "The domain of Arnheim", 536.

${ }^{47}$ Edgar Allan Poe, "The poetic principle", en The complete works of Edgar Allan Poe, ed. por James A. Harrison (Nueva York/Filadelfia/Chicago: John D. Morris and Company, 1902), XIV, 279. 
centelleando en el rojo poniente como región de los muertos evocada por el color de la sangre del atardecer, el aspecto fantasmal de la construcción y los espíritus (entre silfos, hadas, genios y gnomos) que, se dice, parecen haberlo creado. En efecto, como se indica en el texto, el edificio es, si no una tumba, sí un recuerdo fúnebre de Ellison, su rico propietario ya difunto para ese momento, presuntamente asesinado por ciertos visitantes del parque que no son caracterizados: "the late death of my friend, in causing his domain to be thrown open to certain classes of visitor, has given to Arnheim a species of secret and subdued if not solemn celebrity, similar in kind, although infinitely superior in degree, to that which so long distinguished Fonthill". ${ }^{48}$

Con esta referencia, el edificio, descrito en el relato como de hechura semigótica, semiárabe, podría estar inspirado en Fonthill Abbey, gran casa de campo construida en estilo neogótico (igual que los cottages propuestos por A.J. Downing) por William Thomas Beckford a finales del siglo XVIII en Wiltshire, Inglaterra, cuyo trágico y romántico derrumbe era bien conocido en tiempos de Poe, mientras que la "cierta clase de visitantes" podría referirse a los homosexuales de los que, según voz popular, se

\footnotetext{
${ }^{48}$ Poe, "The domain of Arnheim", 535.
}

rodeaba Bedford y que habían encontrado en él un patrocinador y un compañero, pero que al mismo tiempo lo habían exiliado del contacto de las personas honorables de su nivel, como si estuviera muerto para la sociedad.

Paralelismos e inspiraciones al margen, todo ello sirve de pretexto a Poe para explicar su concepción del orden del mundo. Según Poe, es posible que todo lo que en la tierra luce desordenado y caótico sea orden y regularidad para un observador externo, concretamente los ángeles terrenales o espíritus de los hombres muertos, invisibles para la humanidad, con una apreciación de la belleza refinada por la muerte, ${ }^{49}$ para quienes Dios dispuso la visión del jardín terrenal en su panorámica más amplia y hermosa. De esta manera, si el hombre quisiera percibir la verdadera belleza unitaria del mundo debería hacerlo desde lo alto, como aquellos espíritus,

to take such a survey of the universe that the mind may be able really to receive and to perceive an individual impression.

He who from the top of Etna casts his eyes leisurely around, is affected chiefly by the extent and diversity of the scene. Only by a rapid whirling on his heel could he hope to comprehend the panorama in the sublimity of its oneness. ${ }^{50}$

${ }^{49}$ Ibid., 532.

${ }^{50}$ Poe, Eureka. A prose poem, 8. 
La perfección unitaria de un paisaje pasaría así a convertirse en una cuestión de perspectiva, y como lo que a los vivos les interesa es imitar la belleza superior percibida por los ángeles desde el cielo, tienen que recurrir a las matemáticas para encontrar en la disposición y las proporciones de los elementos del paisaje terrestre en pequeño lo que no pueden alcanzar con sus limitadas visiones a ras de suelo, esto es: volviéndolo moral por medio de un orden comprensible para el ser humano y para lo cual es imprescindible acortar las distancias desde las que se mira, pues lo que observado en general o desde mayor distancia en la naturaleza, como lo hacen los ángeles, podría considerarse un detalle positivo, observado de cerca por los hombres podría causar en ellos un pésimo efecto ${ }^{51}$ al perder la visión completa en la cual las partes tienen sentido. Por eso, a lo largo de su narración Poe insiste tanto en que el viajero vaya pasando de un escenario a otro, creados todos ellos en proporción a la capacidad sensorial del hombre como universos coherentes desvinculados unos de los otros y ajenos a la idea de complementariedad con respecto a cualquier cosa.

Entendido lo anterior, queda claro que más que hacia la muerte como una mera extinción de la conciencia,

${ }^{51}$ Poe, "The domain of Arnheim", 10. según se antoja en una lectura más superficial del texto, el viaje lleva en realidad hacia un intento de percepción del mundo similar a la divina de un paisaje perfeccionado matemáticamente por el hombre por medio del recurso de la fragmentación, a imagen del que espera disfrutar como totalidad desde una perspectiva superior en el paraíso, lo cual acerca también metafóricamente a los hombres con los ángeles mismos. Sin embargo, lejos de los ambientes vaporosos, indefinidos y tenues que luego veríamos en las obras de Lord Dunsany ${ }^{52}$ (imitadas por H.P. Lovecraft en sus primeros amagos de escritor), a medio camino entre el verdadero sueño y la alucinación, en su racionalismo, Poe no acierta a crear un espacio mágico sin hacerlo participar de la pesadez grosera del mundo material. Su jardín perfecto sigue siendo un jardín humano, un parque de diversiones con toda la tramoya que el dinero puede comprar, pero que no por deslumbrante resulta menos mecánico con las impresionantes obras hidráulicas que empujan la canoa de las visitas, así como los efectos musicales y odoríferos que se despliegan al final del camino con las puertas gigantescas que se abren de manera automática a su paso y que, paradójicamente, lo hacen

${ }^{52}$ Edward JMD Plunkett, $18^{\circ}$ Baron de Dunsany (1878-1957). Su obra más conocida es $L a$ hija del rey de las hadas. 
exclamar que todo aquello era "como un sueño". ${ }^{33}$

Y tal vez fuera en verdad un sueño, pero en todo caso era el sueño de Herón de Alejandría (inventor griego del siglo I d.C. a quien Poe ya había citado explícitamente en el cuento "Conversación con una momia") con sus puertas de apertura automática movidas por agua que, al dejar escapar la presión de un "molino de viento" hacían sonar simultáneamente órganos musicales escondidos y ponían a gorjear pájaros mecánicos entre las frondas. ${ }^{54}$ Un sueño tecnológico de resortes, contrapesos y ruedas dentadas que se mueven con precisión y regularidad cumpliendo su cometido sobre un paisaje que imita la vida sin engañar a nadie en su intento de presentar un orden perfecto y un paraíso idealizado. A pesar de su estilizada y romántica forma de comprender el orden del mundo, su paraíso sobre la tierra no era más que bella chatarra en movimiento.

Como ocurre en su ensayo "La filosofía de la composición” (1846), todo en los jardines y paisajes de Poe es formalismo, una serie de estructuras de gran belleza y espiritualidad aparente, pero que por debajo de sus prodigios de inventio y de poesis se manifiestan como creaciones cere-

${ }^{53}$ Poe, "The domain of Arnheim", 23.

${ }^{54}$ Heronis Alexandrini, Opera quae supersunt omnia, vol. I (Leipzig: B. G. Teubner, 1899). brales meticulosamente planeadas y llevadas a cabo sin espacio para la improvisación.

\section{Conclusiones}

Para Poe el mundo no era un caos inextricable, sino un universo con dos distintas realidades: pesada y cotidiana una, espiritual y extraordinaria la otra (en el sentido dado por Poe a lo espiritual como materia imparticulada y no simplemente como una materia enrarecida al extremo), ${ }^{55}$ y creía que si un principio de desorden y de confusión se halla instalado en el universo este procede de la contingencia, anarquía e historicidad del mundo físico, el cual requiere un aliño artificial que le de una estabilidad similar al mundo espiritual, en donde existe un orden supramundano perfecto, atemporal y necesario.

Si bien es en los pasajes filosóficos, religiosos y cosmogónicos de sus textos donde Poe expone sus vislumbres acerca del orden absoluto del mundo, es en los ensayos de "interiorismo" y de "jardinería" donde puede verse su interés práctico en imponerle reglas al medio, planteando las relaciones perfectas de proporción, distribución, color y perspectiva necesarias para alcanzar la armonía

\footnotetext{
${ }^{55}$ Poe, "Mesmeric revelation", 45-46.
} 
suprema que le faltan en la experiencia cotidiana de los seres humanos. Experiencia en donde el caos o, mejor, la sensación de caos, se deriva precisamente de la incapacidad del hombre común para entender las reglas y los mecanismos del mundo que le rodea, y para cuya corrección se requería, según Poe, el uso de un doble método que postulaba por un lado el arma todo poderosa de las matemáticas, mientras que por el otro (con las limitaciones del buen sentido), confiaba en la capacidad intuitiva de los individuos "de genio" para usar las regularidades y las excepciones encontradas en el libro de la naturaleza como indicios para su comprensión. Como afirma Leon Howard: sus relatos "de sensación y raciocinio no reflejan ningún serio conflicto entre la sensibilidad y la racionalidad de Poe. En ellos cada cualidad de su mente era usada para reforzar la otra". ${ }^{56}$

Para Poe, como para los trascendentalistas, la comprensión de la vida solo podía pasar por la comprensión del universo, pues las leyes absolutas de uno parecían darle estabilidad a la otra salvándola de su inaceptable contingencia. Sin embargo, a diferencia de los trascendentalistas, todo sentimiento y misticismo, que se conformaban con experimentar la naturaleza confiando que así experimentaban a Dios, y a pesar de todo el valor que Poe le confería a la intuición, nuestro autor no podía sentir ese contacto ni evitar darse cuenta de los defectos del fragmentario mundo inmediato pese al presentimiento de la belleza trascendente del mismo como unidad. Fuera del papel, su explicación del universo no le servía a Poe para solucionar su precaria existencia real ni crear un entorno como él hubiera deseado para su familia. Sus matemáticas no le ayudaban, su caricatura panenteísta del mundo no le daba una esperanza ni le evitaba ser para muchos de sus contemporáneos más que un pobre diablo y un inadaptado.

${ }^{56}$ Leon Howard, "Artificial sensitivity and artful rationality: Basic elements in the creative imagination of Edgar Allan Poe", Poe Studies / Dark Romanticism 20 (1987), 5. 